\title{
Twistor Theory of the Airy Equation ${ }^{\star}$
}

Michael COLE and Maciej DUNAJSKI

Department of Applied Mathematics and Theoretical Physics, University of Cambridge, Wilberforce Road, Cambridge CB3 OWA, UK

E-mail:m.dunajski@damtp.cam.ac.uk

Received November 28, 2013, in final form March 18, 2014; Published online March 29, 2014

http://dx.doi.org/10.3842/SIGMA.2014.037

Abstract. We demonstrate how the complex integral formula for the Airy functions arises from Penrose's twistor contour integral formula. We then use the Lax formulation of the isomonodromy problem with one irregular singularity of order four to show that the Airy equation arises from the anti-self-duality equations for conformal structures of neutral signature invariant under the isometric action of the Bianchi II group. This conformal structure admits a null-Kähler metric in its conformal class which we construct explicitly.

Key words: twistor theory; Airy equation; self-duality

2010 Mathematics Subject Classification: 32L25; 34M56

\section{Introduction}

The Airy equation

$$
f(t)^{\prime \prime}+t f(t)=0
$$

admits solutions given by integrals

$$
f(t)=\int_{\Gamma} \exp \left(\frac{1}{3} \lambda^{3}+\lambda t\right) d \lambda
$$

where $\lambda \in \mathbb{C}$ is an auxiliary complex parameter (see, e.g., [4]). Two linearly independent solutions correspond to open contours $\Gamma=\Gamma_{1}$ and $\Gamma=\Gamma_{2}$ in the complex plane, each of which begins in one of the three shaded sectors where $\cos (3 \arg (\lambda))<0$ and ends in another such sector (Fig. 1).

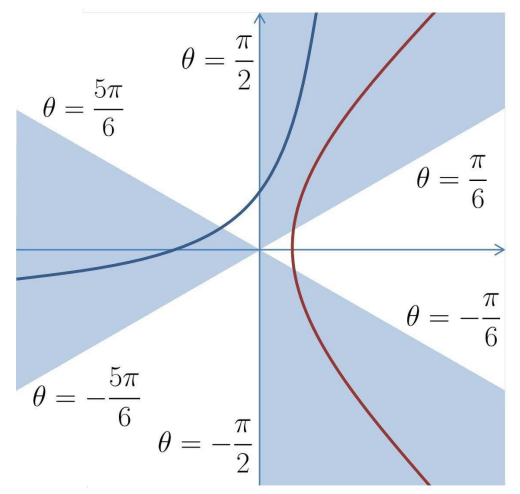

Figure 1. Contours in Airy's formula start and end in the shaded sectors.

${ }^{\star}$ This paper is a contribution to the Special Issue on Progress in Twistor Theory. The full collection is available at http://www.emis.de/journals/SIGMA/twistors.html 
This observation can be verified by differentiating inside the integral and integrating by parts. The boundary term is then required to vanish which restricts the allowed form of the contours.

Airy's formula (2) is reminiscent of Penrose's twistor contour integral formula [9] for the solutions to the wave equation on the complexified Minkowski space $\mathbb{C M}=\mathbb{C}^{4}$. If $(w, z, \tilde{w}, \tilde{z})$ are holomorphic coordinates on $\mathbb{C M}$ such that the line element is $d s^{2}=d z d \tilde{z}-d w d \tilde{w}$, then all solutions of the wave equation

$$
\phi_{z \tilde{z}}-\phi_{w \tilde{w}}=0
$$

are of the form

$$
\phi(w, z, \tilde{w}, \tilde{z})=\oint_{\Gamma} \psi(w+\lambda \tilde{z}, z+\lambda \tilde{w}, \lambda) d \lambda,
$$

where $\Gamma \subset \mathbb{C P}^{1}$ is a closed contour, and the function $\psi$ is holomorphic in $\lambda \in \mathbb{C P}^{1}$ except some singularities inside $\Gamma$.

In Section 2 we shall demonstrate that (2) is a special case of (4). Our procedure is an application of symmetry reductions [7] which have previously been used to analyse non-linear ODEs arising from a non-abelian generalisation of (3). Our result emphasises the cohomological nature of twistor integral formulae: the general solution to (3) depends on two arbitrary functions of three variables, and yet the twistor formula (4) contains only one such function on the r.h.s. How is that possible? The answer - hidden in the abstract machinery of the Penrose transform [3] is that solutions to (3) correspond to the equivalence classes $(\Gamma, \psi)$ of contours and cohomology representatives rather than (naively) to holomorphic functions of three variables. The example of the Airy equation makes this explicit: the general solution to the linear ODE (1) depends on two constants of integration, and is a linear combination of two linearly independent solutions. Both solutions are of the form (2) with the same integrand (which plays a role of the twistor function $\psi$ ), but different contours.

In Section 3 we shall show that the Airy equation also arises from the anti-self-duality equations on a cohomogeneity one-conformal structure of neutral signature invariant under the Bianchi II group. This conformal structure admits a null-Kähler metric in its conformal class which we construct explicitly. The twistor distribution defining the $\alpha$-surfaces is equivalent to the Lax pair of [5] for the isomonodromic problem with one irregular singularity of order four.

\section{Construction of the twistor function}

Let us start from the following lemma which can be proven by explicit calculation:

Lemma 1. Set $t=\tilde{z}-z-\tilde{w}^{2}$. The function

$$
\phi(w, z, \tilde{w}, \tilde{z})=\exp \left(w-\tilde{w}(z-\tilde{z})-\frac{2}{3} \tilde{w}^{3}\right) f(t)
$$

satisfies the wave equation (3) if and only if $f(t)$ is a solution to the Airy equation (1).

This result is valid both in the holomorphic and real category. In the latter case all the coordinates are assumed to be real, and $\phi$ satisfies the ultra-hyperbolic wave equation on $\mathbb{R}^{2,2}[6,14]$. The geometric interpretation of Lemma 1 is as follows: The wave equation on $\mathbb{C M}$ is conformally invariant, and the conformal group of the complexified Minkowski space is $\operatorname{SL}(4, \mathbb{C})$. The special solutions (5) are invariant under the action of the three-dimensional abelian subgroup $H$ of $\mathrm{SL}(4, \mathbb{C})$ generated by the vector fields

$$
X=\partial_{w}, \quad Y=\partial_{z}+\partial_{\tilde{z}}, \quad Z=(z-\tilde{z}) \partial_{w}+\tilde{w}\left(\partial_{\tilde{z}}-\partial_{z}\right)+\partial_{\tilde{w}},
$$


and invariance condition takes the form

$$
X(\phi)=\phi, \quad Y(\phi)=0, \quad Z(\phi)=0 .
$$

The readers familiar with the twistor approach to the Painlevé equations [7] will recognise this abelian group. A symmetry reduction of the anti-self-dual Yang-Mills equation by $H$ yields the Painlevé II equation. This is not unexpected, as from the isomonodromic perspective both the Airy equation and the Painlevé II correspond to an irregular singular point of order four.

To proceed further we recall the basic twistor correspondence and the double fibration picture (see, e.g., $[2,7]$ ). The points of the three-dimensional twistor space $\mathbb{P T}=\mathbb{C P}^{3}-\mathbb{C P}^{1}$ are $\alpha$ planes in $\mathbb{C M}$ : two-dimensional planes which are totally null with respect to the line element $d s^{2}$, and such that their tangent bi-vector is self-dual with respect to a holomorphic volume form $d w \wedge d \tilde{w} \wedge d z \wedge d \tilde{z}$. There is a rational curve $L_{p} \cong \mathbb{C P}^{1}$ worth of such planes through each point $p \in \mathbb{C M}$, and so points in $\mathbb{C M}$ correspond to rational curves in $\mathbb{P T}$. The twistor space arises as a quotient of the five-dimensional correspondence space $\mathcal{F}=\mathbb{C M} \times \mathbb{C P}^{1}$ by a two-dimensional integrable distribution spanned by the vector fields

$$
l=\lambda_{0} \partial_{\tilde{z}}-\lambda_{1} \partial_{w}, \quad m=\lambda_{0} \partial_{\tilde{w}}-\lambda_{1} \partial_{z},
$$

where $\left[\lambda_{0}, \lambda_{1}\right]$ are homogeneous coordinates of a point in $\mathbb{C P}^{1}$. For each fixed $\left[\lambda_{0}, \lambda_{1}\right] \in \mathbb{C P}^{1}$ these vector fields span an $\alpha$-plane through a point in $\mathbb{C M}$. A twistor function is a function on $\mathcal{F}$ which is constant along $l$ and $m$. In a patch containing $[1,0] \in \mathbb{C P}^{1}$ define $\lambda=\lambda_{1} / \lambda_{0}$. The local coordinates on $\mathbb{P T}$ pull back to three twistor functions

$$
\mu=w+\lambda \tilde{z}, \quad \nu=z+\lambda \tilde{w}, \quad \lambda .
$$

Fixing $(w, z, \tilde{w}, \tilde{z})$ in $(7)$ gives a rational curve in $\mathcal{F}$ which descends down to $\mathbb{P} \mathbb{T}$. Conversely, fixing $(\lambda, \mu, \nu)$ in $(7)$ gives an $\alpha$-plane in $\mathbb{C M}$.

To arrive at the contour formula (4) consider a twistor function $\psi(\mu, \nu, \lambda)$ defined on an intersection of two open sets containing $\lambda_{0}=0$ and $\lambda_{1}=0$ respectively. Now restrict $\psi$ to a twistor line $L_{p} \cong \mathbb{C P}^{1}$, and integrate its pull back to $\mathcal{F}$ over a closed contour in the pre-image of $L_{p}$ in $\mathcal{F}$. This gives a function at $p \in \mathbb{C M}$ which satisfies the wave equation (3). The volume element $d \lambda$ on $L_{p}$ is a section of a line bundle $\mathcal{O}(2) \rightarrow L_{p}$, so for all of this to work $\psi$ must really be a representative of a cohomology class $H^{1}\left(L_{p}, \mathcal{O}(-2)\right)$.

To implement the symmetry condition we need to consider the action of $H$ on $\mathcal{F}$, where in addition to moving points in $\mathbb{C M}$ it also changes the $\alpha$-planes through a point. This is done by constructing lifts $\left(X^{\prime \prime}, Y^{\prime \prime}, Z^{\prime \prime}\right)$ of the generators $(6)$ to $\mathcal{F}$ such that the resulting vector fields commute with $l$ and $m$ modulo $(l, m)$. The lifted vector fields are

$$
X^{\prime \prime}=X, \quad Y^{\prime \prime}=Y, \quad Z^{\prime \prime}=Z+\partial_{\lambda} .
$$

They push forward to holomorphic vector fields $\left(X^{\prime}, Y^{\prime}, Z^{\prime}\right)$ on the twistor space, which can be constructed by an application of the chain rule. This yields

$$
X^{\prime}=\partial_{\mu}, \quad Y^{\prime}=\partial_{\nu}+\lambda \partial_{\mu}, \quad Z^{\prime}=\nu \partial_{\mu}+\lambda \partial_{\nu}+\partial_{\lambda}
$$

Now set $\Omega=\psi(\mu, \nu, \lambda) d \lambda$, and impose the symmetry condition

$$
\mathcal{L}_{X^{\prime}} \Omega=\Omega, \quad \mathcal{L}_{Y^{\prime}} \Omega=0, \quad \mathcal{L}_{Z^{\prime}} \Omega=0
$$

where $\mathcal{L}$ is the Lie derivative. This yields

$$
\Omega=\exp \left(\mu-\lambda \nu+\lambda^{3} / 3\right) d \lambda=\exp \left(w-\tilde{w}(z-\tilde{z})-\frac{2}{3} \tilde{w}^{3}\right) \exp \left(\frac{1}{3}(\lambda-\tilde{w})^{3}+(\lambda-\tilde{w}) t\right) d \lambda
$$




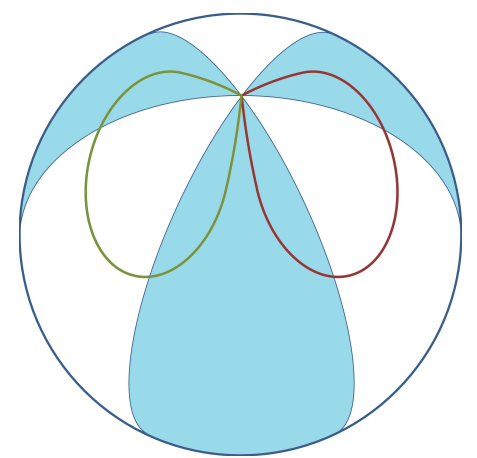

Figure 2. Closed contours in the Riemann sphere corresponding to a point in $\mathbb{C M}$.

up to an overall constant multiple, where in the second line above we have restricted $\Omega$ to the twistor line (7). Performing a Möbius transformation $\lambda \rightarrow(\lambda+\tilde{w})$ and using (5) reduces the Penrose formula (4) to the Airy formula (2). The contours from Fig. 1 are now re-interpreted as closed contours in the Riemann sphere $L_{p} \subset \mathbb{P T}$ corresponding to $p \in \mathbb{C M}$. The twistor function $\psi$ has an essential singularity at the pole $\lambda=\infty$ which belongs to both contours. The integrals are nevertheless well defined as $\psi$ approaches the singularity in the shaded sectors.

\section{ASD conformal structure with Bianchi II symmetry}

In this section we shall give an alternative twistor construction for the Airy equation, this time using this equation to construct a conformal class of curved metrics in four dimensions with anti-self-dual Weyl tensor.

The nonlinear graviton construction on Penrose [10] extends twistor theory to curved backgrounds. An $\alpha$-surface in an oriented holomorphic four-manifold $M$ with a holomorphic metric $g$ is a two-dimensional surface $\xi$ such that $T_{p} \xi$ is an $\alpha$-plane for any $p \in \xi$. A seminal result of [10] is that there locally exist a three-parameter family of $\alpha$-surfaces iff the self-dual part of the Weyl tensor of $g$ vanishes. We then say that the conformal structure $[g]:=\left\{\Omega g, \Omega: M \rightarrow \mathbb{R}^{+}\right\}$ is anti-self-dual (ASD). A twistor space $\mathbb{P T}$ of an ASD four-manifold is defined to be a space of $\alpha$-surfaces. It is a three-dimensional complex manifold with a four-parameter family of rational curves with normal bundle $\mathcal{O}(1) \oplus \mathcal{O}(1)$. The conformal structure of $g$ is encoded in the complex structure of the twistor space, which for non-conformally flat ASD manifolds is different than that of $\mathbb{C P}^{3}-\mathbb{C P}^{1}$. A convenient way to express the ASD condition on a conformal structure is summarised in the following theorem $[2,7]$.

Theorem 1. Let $Z, W, \widetilde{Z}, \widetilde{W}$ be four independent holomorphic vector fields on $M$. The conformal structure defined by

$$
g=Z \odot \widetilde{Z}-W \odot \widetilde{W}
$$

is $A S D$ if and only if there exists functions $f_{0}, f_{1}$ on $M \times \mathbb{C P}^{1}$ cubic in $\lambda \in \mathbb{C P}^{1}$ such that the distribution

$$
l=\widetilde{Z}-\lambda W+f_{0} \frac{\partial}{\partial \lambda}, \quad m=\widetilde{W}-\lambda Z+f_{1} \frac{\partial}{\partial \lambda}
$$

is Frobenius integrable, i.e. $[l, m]=0$ modulo $l$ and $m$.

If the integrability condition holds then there is a $\mathbb{C P}^{1}$-worth of $\alpha$-surfaces spanned by $\{\widetilde{Z}$ $\lambda W, \widetilde{W}-\lambda Z\}$ through any point in $M$. If all vectors $(Z, \ldots, \widetilde{W})$ are real then the signature of $g$ is $(2,2)$, and there exists an $\mathbb{R P}^{1}$-worth of real $\alpha$-surfaces through each point. 
From now on we shall additionally assume that there exists a three-dimensional Lie group $G$ acting on $M$ by conformal isometries with generically three-dimensional orbits. The conformal isometries are generated by the right-invariant vector fields $R_{j}, j=1,2,3$ on $G$. The $G$ action on $M$ maps $\alpha$-surfaces to $\alpha$-surfaces and thus gives rise to a group action of $G$ on the twistor space $\mathbb{P T}$. We can choose to work in an invariant frame, where the lifts of $R_{j}$ s to the correspondence space are given by $R_{j}$ s. Consider a quartic

$$
s=(d \lambda \wedge \operatorname{vol})\left(l, m, R_{1}, R_{2}, R_{3}\right),
$$

where vol is the holomorphic volume form on $M$ such that $\operatorname{vol}(W, \widetilde{W}, Z, \widetilde{Z})=1$. To make contact with the isomonodromic problem for the Airy equation we shall assume that $s$ vanishes at one value of $\lambda$ to order four. We can write the null tetrad of Theorem 1 terms of the vector field $\partial_{t}$ orthogonal to the $G$-orbits, and three linearly independent vector fields $P, Q, R$ tangent to $G$ which are $t$-dependent and invariant under left translations:

$$
\widetilde{Z}=\partial_{t}+R, \quad Z=\partial_{t}-R, \quad W=-P, \quad \widetilde{W}=Q .
$$

Moreover the invariance condition implies that $f_{0}$ and $f_{1}$ are constant on $G$, and so depend only on $\lambda$ and $t$. A direct calculation now shows that the quartic $s$ is proportional to $\left(\lambda f_{0}+f_{1}\right)$. This quartic has a quadrupole zero which we shall move to $\lambda=\infty$ by a Möbius transformation. Using the freedom in the left and right rotations of the tetrad, and the ASD equations it is possible to set $\left(f_{0}=0, f_{1}=1\right)$. Now consider ${ }^{1}$ a pair of linear combinations of $l$ and $m(9)$ given by

$$
L:=\frac{\lambda l+m}{\lambda f_{0}+f_{1}}=\frac{\partial}{\partial \lambda}+Q+2 \lambda R+\lambda^{2} P, \quad M:=\frac{f_{1} l-f_{0} m}{\lambda f_{0}+f_{1}}=\frac{\partial}{\partial t}+R+\lambda P .
$$

Since the conformal class is ASD, Theorem 1 implies that $[L, M]=0$, modulo $L$ and $M$. However $[L, M]$ does not contain $\partial_{\lambda}$ or $\partial_{t}$, thus $[L, M]$ must be identically zero which yields

$$
Q^{\prime}=[Q, R]+P, \quad R^{\prime}=\frac{1}{2}[Q, P], \quad P^{\prime}=[R, P] .
$$

We shall now make a choice for $G$, and take it to be the Bianchi II group. Its Lie algebra is generated by the left invariant vector fields $L_{j}$ on $G$ which satisfy

$$
\left[L_{1}, L_{2}\right]=L_{1}, \quad\left[L_{1}, L_{3}\right]=0, \quad\left[L_{2}, L_{3}\right]=0
$$

The connection with the Airy equation is provided by the following result.

Theorem 2. A general cohomogeneity-one Bianchi II ASD conformal class such that the twistor quartic (10) admits a zero of order four is of the form (8), (11), with

$$
\begin{aligned}
P & =\frac{1}{2} F L_{1}+L_{2}, \\
Q & =\left(\frac{1}{2}\left(c_{1}^{2}+t\right) F+c_{1} F^{\prime}+F^{\prime \prime}\right) L_{1}+\left(c_{1}^{2}+t\right) L_{2}+c_{2} L_{3}, \\
R & =\frac{1}{2}\left(c_{1} F+F^{\prime}\right) L_{1}+c_{1} L_{2}+L_{3},
\end{aligned}
$$

where $\left(c_{1}, c_{2}\right)$ are constants, and $f(t)=F^{\prime}(t)$ satisfies the Airy equation (1).

\footnotetext{
${ }^{1}$ In [8] it was instead assumed that the quartic $s$ has four distinct zeros, and that $G=\operatorname{SL}(2, \mathbb{C})$ which lead to the isomonodromic Lax pair [5] for Painlevé VI.
} 
Proof. To complete the proof we need to show that any solution to the reduced ASD equations (12) can be put in the form (2) without the loss of generality. The three vector fields $P$, $Q, R$ can be written in the basis of left-invariant vector fields $L_{1}, L_{2}, L_{3}$ satisfying (13) as

$$
\begin{aligned}
& P=p_{1}(t) L_{1}+p_{2}(t) L_{2}+p_{3}(t) L_{3}, \\
& Q=q_{1}(t) L_{1}+q_{2}(t) L_{2}+q_{3}(t) L_{3}, \\
& R=r_{1}(t) L_{1}+r_{2}(t) L_{2}+r_{3}(t) L_{3},
\end{aligned}
$$

for some functions $p_{j}(t), q_{j}(t), r_{j}(t)$. The Lie algebra relation (13) is preserved by

$$
L_{1} \rightarrow \alpha L_{1}, \quad L_{2} \rightarrow L_{2}+\beta L_{3}, \quad L_{3} \rightarrow \gamma L_{3},
$$

where $(\alpha \neq 0, \beta, \gamma \neq 0)$ are constants. The third equation in (12) implies that $p_{2}=c_{0}$ is a constant and that $p_{3}$ is a constant which can be set to zero using (15). We also get $p_{1}^{\prime}=r_{1} p_{2}-p_{1} r_{2}$. Let us set $p_{1}=F / 2$, where $F=F(t)$. The second equation in (12) implies that $r_{2}=c_{1}$ is a constant and that $r_{3}$ is another constant which we can set to 1 using (15). We also obtain

$$
q_{1}=\frac{2 r_{1}{ }^{\prime}}{c_{0}}+\frac{q_{2} F}{2 c_{0}} .
$$

Finally the first equation in (12) implies that $q_{3}=c_{2}$ is a constant, $q_{2}=c_{0} t+c_{3}$, where $c_{3}$ is another constant, and it also gives

$$
F^{\prime \prime \prime}+\left(\left(c_{3} c_{0}-c_{1}^{2}\right)+t c_{0}^{2}\right) F^{\prime}=0 .
$$

The result now follows by making an affine transformation of $t$ to set $c_{0}=1, c_{3}=c_{1}^{2}$.

We shall end this section discussing the connection between the system (12) and the isomonodromic problem with irregular singularity of order four. Consider a $2 \times 2$ matrix

$$
\Theta(t, \lambda)=Q+2 \lambda R+\lambda^{2} P
$$

where $\lambda \in \mathbb{C P}^{1}$, and $P, Q, R$ are elements of a matrix Lie algebra $\mathfrak{g}$ which also depend on a parameter $t$. For a chosen fixed value of $t$ consider a linear matrix ODE

$$
\frac{d \Psi}{d \lambda}+\Theta \Psi=0
$$

Now allow $t$ to vary on the complex plane, so that the matrix fundamental solution $\Psi$ depends on $\lambda$ and $t$. The monodromy around the fourth-order pole $\lambda=\infty$ does not depend on $t$ if $\Psi$ satisfies [5]

$$
\frac{\partial \Psi}{\partial \lambda}+\Theta \Psi=0, \quad \frac{\partial \Psi}{\partial t}+\Theta_{+} \Psi=0, \quad \text { where } \quad \Theta_{+}:=R+\lambda P .
$$

The compatibility conditions for this overdetermined linear system reduce to system of nonlinear matrix $\operatorname{ODEs}^{2}(12)$ for $(P, Q, R)$. If $P$ is diagonalisable, and $\mathfrak{g}=\mathfrak{s l}(2, \mathbb{C})$, then (12) reduce to Painlevé II. Theorem 2 shows that if instead $\mathfrak{g}$ is the Bianchi II algebra (13) then the isomonodromic condition is the (derivative of) the Airy equation.

${ }^{2}$ These compatibility conditions are

$$
\frac{1}{2} \frac{\partial^{2} \Theta}{\partial \lambda^{2}}-\frac{\partial \Theta}{\partial t}+\frac{1}{2}\left[\Theta, \frac{\partial \Theta}{\partial \lambda}\right]=0 .
$$

Rescaling $\partial / \partial t \rightarrow \epsilon \partial / \partial t$ and $\partial / \partial \lambda \rightarrow \epsilon \partial / \partial \lambda$, and taking the dispersionless limit $\epsilon \rightarrow 0$ yields the Nahm equations for $(P, Q, R)$. In this limit the spectral curve $S=\left\{(\omega, \lambda) \in T \mathbb{C P}^{1} \mid \operatorname{det}(\mathbf{1} \omega-\Theta(t, \lambda))=0\right\}$ does not depend on $t$. This is in agreement with the observation of [12] that isospectral deformations arise as a limit of isomonodromic deformations. 


\subsection{ASD null-Kähler structure}

A null-Kähler structure on a four-dimensional manifold $M$ is a pair $(\hat{g}, N)$ where $\hat{g}$ is a metric of signature $(2,2)$ and $N: T M \longrightarrow T M$ is a rank-2 endomorphism such that

$$
N^{2}=0, \quad \hat{g}(N X, Y)+\hat{g}(X, N Y)=0, \quad \nabla N=0
$$

for all vector fields $X, Y$ on $M$. Given such $N$ and $\hat{g}$ we can construct a null-Kähler two-form $\Sigma$ such that $\Sigma(X, Y)=\hat{g}(N X, Y)$ and

$$
\nabla \Sigma=0, \quad \Sigma \wedge \Sigma=0
$$

In [1] it was shown that a null-Kähler structure gives rise to a preferred section of $\kappa^{-1 / 4}$, where $\kappa$ is the holomorphic canonical bundle of the twistor space, and conversely given that $\mathbb{P T}$ admits such an anti-canonical divisor the corresponding ASD conformal class admits a null-Kähler structure. The conformal class defined by $(P, Q, R)$ from Lemma 2 gives rise to a section of $\kappa^{-1 / 4}$ given by a push-forward of the quartic (10) to $\mathbb{P T}$ vanishes at each twistor line at one point, where the holomorphic vector fields corresponding to the isometries become linearly dependent. This conformal class should therefore contain a null-Kähler structure. To construct it explicitly, choose the coordinates $(x, y, z)$ on $G$ such that the left-invariant vector fields are

$$
L_{1}=e^{-y} \partial_{x}, \quad L_{2}=\partial_{y}, \quad L_{3}=\partial_{z} .
$$

To simplify the formulae, we shall also choose $c_{1}=c_{2}=0$ in (2). This yields the basis of one-forms dual to $(Z, W, \widetilde{Z}, \widetilde{W})$ given by

$$
\begin{aligned}
\mathbf{e}_{\widetilde{Z}} & =\frac{1}{2}(d t+d z), & \mathbf{e}_{\widetilde{W}} & =\frac{1}{F^{\prime \prime}}\left(e^{y} d x-\frac{1}{2} F d y-\frac{1}{2} F^{\prime} d z\right), \\
\mathbf{e}_{Z} & =\frac{1}{2}(d t-d z), & \mathbf{e}_{W} & =\frac{1}{F^{\prime \prime}}\left(t e^{y} d x-\left(\frac{t}{2} F+F^{\prime \prime}\right) d y-\frac{t}{2} F^{\prime} d z\right) .
\end{aligned}
$$

The conformally rescaled metric

$$
\hat{g}=\Omega\left(\mathbf{e}_{Z} \odot \mathbf{e}_{\widetilde{Z}}-\mathbf{e}_{W} \odot \mathbf{e}_{\widetilde{W}}\right), \quad \text { where } \quad \Omega=e^{-y}\left(F^{\prime \prime}\right)
$$

is ASD and null-Kähler with the null-Kähler two-form $\Sigma$ given by

$$
\Sigma=\Omega\left(\mathbf{e}_{\widetilde{Z}} \wedge \mathbf{e}_{\widetilde{W}}\right)
$$

This is also gives the null-Kähler two-form in the general case when the constants $\left(c_{1}, c_{2}\right)$ are arbitrary, in which case the conformal factor needs to be replaced by $\Omega=e^{-y}\left(F^{\prime \prime}+\left(c_{1}-c_{2} / 2\right) F^{\prime}\right)$.

\section{Conclusions}

We have shown that the integral formula for the Airy equation is a special case of Penrose's twistor integral formula. Other special functions also admit a twistor description. In particular the hyper-geometric function together with its generalisations have been investigated in [11]. All twistor integral formulae for special functions could presumably be obtained from the results of this work by confluence of singularities of the twistor quartic $\operatorname{vol}_{\mathbb{P T}}\left(X^{\prime}, Y^{\prime}, Z^{\prime}\right)$, where $X^{\prime}, Y^{\prime}, Z^{\prime}$ are holomorphic vector fields on $\mathbb{P T}$ which correspond to the generators of the Painlevé VI abelian subgroup of $\mathrm{SL}(4, \mathbb{C})$. The details of this have not been worked out, but it may be interesting to do so. 
The one-form

$$
A=\phi_{z} d \tilde{w}+\phi_{w} d \tilde{z}
$$

is a solution of the ASD Maxwell equations $F=-* F$, where $F=d A$, if and only if $\phi$ satisfies (3). ASD Maxwell fields correspond, via the Ward transform [13], to holomorphic line bundles over $\mathbb{P T}$ which are trivial on all twistor lines. Thus there is a particular two-parameter class of such line bundles corresponding to the solutions of the Airy equations. These bundles can be characterised by their $H$-invariance along the lines explained in [7].

In Section 3 we have found a neutral signature cohomogeneity-one metric on a four-manifold $M=\mathbb{R} \times G$, where $G$ is the three-dimensional Bianchi II Lie group, such that the anti-selfduality condition on the Weyl tensor reduces to the Airy equation. In this case the twistor distribution $(l, m)$ defining the three-parameter family of $\alpha$-surfaces in $M$ is equivalent to the Lax pair for the isomonodromic problem with one irregular singularity of order four. The resulting metric is conformally related to an ASD null-Kähler structure.

\section{Acknowledgement}

MC would like to thank James Bridgwater for the financial support.

\section{References}

[1] Dunajski M., Anti-self-dual four-manifolds with a parallel real spinor, Proc. Roy. Soc. London Ser. A 458 (2002), 1205-1222, math.DG/0102225.

[2] Dunajski M., Solitons, instantons, and twistors, Oxford Graduate Texts in Mathematics, Vol. 19, Oxford University Press, Oxford, 2010.

[3] Eastwood M.G., Penrose R., Wells R.O., Cohomology and massless fields, Comm. Math. Phys. 78 (1981), 305-351.

[4] Jeffreys H., Jeffreys B.S., Methods of mathematical physics, Cambridge University Press, New York, 1946.

[5] Jimbo M., Miwa T., Monodromy preserving deformation of linear ordinary differential equations with rational coefficients. II, Phys. D 2 (1981), 407-448.

[6] John F., The ultrahyperbolic differential equation with four independent variables, Duke Math. J. 4 (1938), 300-322.

[7] Mason L.J., Woodhouse N.M.J., Integrability, self-duality, and twistor theory, London Mathematical Society Monographs. New Series, Vol. 15, The Clarendon Press, Oxford University Press, New York, 1996.

[8] Maszczyk R., Mason L.J., Woodhouse N.M.J., Self-dual Bianchi metrics and the Painlevé transcendents, Classical Quantum Gravity 11 (1994), 65-71.

[9] Penrose R., Solutions of the zero-rest-mass equations, J. Math. Phys. 40 (1969), 38-39.

[10] Penrose R., Nonlinear gravitons and curved twistor theory, Gen. Relativity Gravitation 7 (1976), 31-52.

[11] Shah M.R., Woodhouse N.M.J., Painlevé VI, hypergeometric hierarchies and Ward ansätze, J. Phys. A: Math. Gen. 39 (2006), 12265-12269.

[12] Takasaki K., Spectral curves and Whitham equations in isomonodromic problems of Schlesinger type, Asian J. Math. 2 (1998), 1049-1078, solv-int/9704004.

[13] Ward R.S., On self-dual gauge fields, Phys. Lett. A 61 (1977), 81-82.

[14] Woodhouse N.M.J., Contour integrals for the ultrahyperbolic wave equation, Proc. Roy. Soc. London Ser. A 438 (1992), 197-206. 